IASSNS-HEP-98-85

LBNL-42428

RU-97-98

October 1998

hep-ph/9810500

\title{
Solving the Supersymmetric Flavor Problem with Radiatively Generated Mass Hierarchies
}

\author{
Jonathan L. Feng, ${ }^{1}$ Christopher Kolda,${ }^{2}$ and Nir Polonsky ${ }^{3}$ \\ ${ }^{1}$ School of Natural Sciences, Institute for Advanced Study \\ Princeton, NJ 08540, USA \\ 2 Theoretical Physics Group, Lawrence Berkeley National Laboratory \\ University of California, Berkeley, CA 94720, USA \\ ${ }^{3}$ Department of Physics and Astronomy, Rutgers University \\ Piscataway, NJ 08854, USA
}

\begin{abstract}
The supersymmetric flavor problem may be solved if the first and second generation scalars are heavy (with multi-TeV masses) and scalars with large Higgs couplings are light (with sub-TeV masses). We show that such an inverted spectrum may be generated radiatively; that is, from initial conditions where all scalar masses are multi-TeV at some high scale, those with large Higgs couplings may be driven asymptotically to the weak scale in the infra-red. The lightness of third generation scalars is therefore a direct consequence of the heaviness of third generation fermions, and fine-tuning is avoided even though the fundamental scale of the soft supersymmetry breaking parameters is multi$\mathrm{TeV}$. We investigate this possibility in the framework of the usual Yukawa quasi-fixed point solutions. The required high scale boundary conditions are found to be simple and highly predictive. This scenario also alleviates the supersymmetric $C P$ and Polonyi problems.
\end{abstract}




\section{Introduction}

If low-energy supersymmetry (SUSY) is realized in nature, the effective Lagrangian must contain many new mass parameters that explicitly, but softly, break supersymmetry. The requirement that large quadratic divergences not be reintroduced in the electroweak breaking sector is often taken to suggest that these soft SUSY-breaking (SSB) parameters are at the scale $m_{\text {light }} \lesssim 1 \mathrm{TeV}$. On the other hand, stringent flavor changing constraints require that many of the soft scalar masses either be at the scale $m_{\text {heavy }} \sim 10 \mathrm{TeV}$ or fall into highly constrained patterns [1]. The tension between these requirements is the supersymmetric flavor problem.

This problem may be resolved, however, if the scalars have an inverted mass hierarchy relative to the fermions [2, 3, 4]. In such a scenario, the scalars of the first two generations are at the scale $m_{\text {heavy }}$. This highly suppresses supersymmetric contributions to flavor (and $C P$ ) violation involving the first two families, where the constraints are most stringent. At the same time, the scalar partners of the heavy fermions, which interact through large Yukawa couplings with the Higgs bosons, are at the scale $m_{\text {light }}$, avoiding fine-tuning in the Higgs sector. Note that, because the scalars of the first two generations interact very weakly with the Higgs bosons, they may be significantly heavier without destabilizing the gauge hierarchy.

This inverted hierarchy of scalar masses has been analyzed in a number of studies, and it has been argued that it may be created by dynamical mechanisms at high [3, 5] or intermediate [6] energies. The experimental signatures of such scenarios have also been studied. Observable effects of the light supersymmetric particles have been considered in Refs. [7, 8], and the non-decoupling effects of very massive superparticles have been discussed in Refs. [9, 10].

In this paper, we note that there is no a priori need to impose this hierarchy among the scalar masses at some high scale, such as the grand unified theory (GUT) or Planck scale, in order to realize the hierarchy at the weak scale. Instead, we demonstrate that even if all soft scalar masses have multi-TeV values at some high scale boundary, the mass hierarchy may be generated radiatively. In this scheme, for specific ratios of the SSB parameters which we will determine, the third generation scalars are driven to the light scale by large Yukawa couplings. The lightness of third generation scalars and heaviness of third generation fermions are therefore intimately connected, and fine-tuning is avoided, even though the fundamental scale of the SSB parameters (the gravitino mass) is $\sim 10 \mathrm{TeV}$.

We will demonstrate this idea in the context of scenarios in which large Yukawa couplings saturate their infra-red quasi-fixed points (QFPs). In this case, the relevant SSB parameters will be seen to have simultaneous (approximate) zero fixed-points. The required boundary conditions for such fixed points to exist will be seen to be remarkably simple and highly predictive, though also highly constrained [11].1]

\footnotetext{
${ }^{1}$ In a related, but orthogonal, approach to the supersymmetric flavor problem, one may search for models in which scalar mass degeneracy, as opposed to a scalar mass hierarchy, is generated by
} 


\section{Inverted Hierarchy Models}

We first review the constraints on supersymmetric models with inverted scalar mass hierarchies.

A supersymmetric scenario is fine-tuned if there are large cancellations in the conditions for electroweak symmetry breaking:

$$
\begin{aligned}
\frac{1}{2} m_{Z}^{2} & =\frac{m_{H_{d}}^{2}-m_{H_{u}}^{2} \tan ^{2} \beta}{\tan ^{2} \beta-1}-\mu^{2} \\
2 m_{3}^{2} & =\left(m_{H_{u}}^{2}+m_{H_{d}}^{2}+2 \mu^{2}\right) \sin 2 \beta .
\end{aligned}
$$

In these equations, $m_{H_{u}}$ and $m_{H_{d}}$ are the SSB Higgs boson masses, $m_{3}^{2}$ is the soft bilinear scalar coupling of the two Higgs doublets, $\mu$ is the Higgsino mass parameter, and $\tan \beta=\left\langle H_{u}^{0}\right\rangle /\left\langle H_{d}^{0}\right\rangle$ is the usual ratio of Higgs vacuum expectation values. In models with hierarchical scalar masses, these conditions have a number of implications resulting from the fact that the light-heavy scalar mass hierarchy and, hence, the Higgs parameters of Eq. (1) are not stable against radiative corrections [13, 2].

Several of these implications are evident even at one-loop [13. For the soft scalar mass parameter $m_{i}$, the one-loop renormalization group equation is

$$
\begin{aligned}
\left.\frac{d m_{i}^{2}}{d t}\right|_{1-\mathrm{loop}}= & 4 \sum_{a} C_{a}(i) \alpha_{a}\left|M_{a}\right|^{2}-\frac{1}{4 \pi} \sum_{p q} h_{i p q}^{2} A_{i p q}^{2} \\
& -\frac{1}{2} Y_{i} \alpha_{Y} \sum_{j} Y_{j} m_{j}^{2}-\frac{1}{4 \pi} \sum_{p q} h_{i p q}^{2}\left(m_{i}^{2}+m_{p}^{2}+m_{q}^{2}\right),
\end{aligned}
$$

where $t=\ln \left(M_{X}^{2} / Q^{2}\right) / 4 \pi$, and $M_{X}$ is the high scale boundary. The index $a$ runs over gauge groups, $C_{a}(i)$ are quadratic Casimir invariants, [] and $Y$ denotes hypercharge. $M, h$, and $h A$ are gaugino masses, Yukawa couplings, and trilinear scalar couplings, respectively. Summations over scalar indices implicitly include summations over color and weak isospin. In general, of course, the masses need not be flavor diagonal, and one must evolve a general mass matrix. Discussion of the off-diagonal masses and their constraints will be deferred to Sec. 7 .

Each of the four terms of Eq. (2) leads to a constraint for generating and maintaining a scalar mass hierarchy. From the first and second terms, we see that gauginos masses and trilinear scalar couplings must be at the light scale. From the third term, which arises from quartic scalar gauge interactions, it is evident that the hypercharge trace must roughly satisfy $\sum_{j} Y_{j} m_{j}^{2} \lesssim m_{\text {light }}^{2}$. From the fourth, one-loop corrections to light scale masses of the form $h^{2} m_{\text {heavy }}^{2}$ lead to the approximate upper bound $m_{\text {heavy }} \lesssim m_{\text {light }} / h$.

fixed points [12].

${ }^{2}$ For the $\mathrm{U}(1)$ gauge group, $C_{a}=Y^{2}$ for scalars with hypercharge $Y$, and for the $\mathrm{SU}(\mathrm{N})$ gauge groups, $C_{a}=\left(N^{2}-1\right) / 2 N$ for scalars in the fundamental representation. 
Even if the three constraints and upper bound mentioned above are satisfied, two-loop gauge interactions threaten to drive the light scalar masses negative. These two-loop corrections are given by [14]:

$$
\left.\frac{d m_{i}^{2}}{d t}\right|_{2-\text { loop }} \propto \sum_{a} \sum_{j}-t_{a}(j) C_{a}(i) \alpha_{a}^{2} m_{j}^{2},
$$

where $a$ again sums over all gauge groups, $t_{a}=Y^{2}$ for hypercharge, and $t_{a}=\frac{1}{2}$ for fundamentals of $\mathrm{SU}(\mathrm{N})$. To avoid tachyonic states and color-breaking minima, these must be compensated by positive contributions from gaugino masses [2, 15, 16]. This observation leads to lower bounds on gaugino masses that are most stringent in models with high-energy mediation of the heavy SSB parameters, where the evolution interval (the logarithm) is maximized.

\section{Radiative Hierarchy with Low $\tan \beta$}

Now we present a first concrete example of the generation of an inverted hierarchy through renormalization group evolution. This will serve as a simple illustration of the idea. A more complicated, but more satisfactory, scenario will be discussed in the following section.

We will consider the minimal supersymmetric standard model (MSSM) with superpotential

$$
W=h_{u}^{i j} H_{u} Q_{i} U_{j}+h_{d}^{i j} H_{u} Q_{i} D_{j}+h_{e}^{i j} H_{d} L_{i} E_{j}+\mu H_{u} H_{d}
$$

where $H_{u}$ and $H_{d}$ are the up- and down-type Higgs superfields, $Q$ and $L$ are the quark and lepton doublets, $U, D$, and $E$ are the up-type quark, down-type quark, and charged lepton singlets, respectively, and the indices $i$ and $j$ denote generations.

We begin by considering the case of low $\tan \beta$. In this scenario, the only significant Yukawa coupling is the top quark Yukawa $h_{t} \equiv h_{u}^{33}$. As noted above, the stability of light-heavy scalar hierarchies requires gaugino masses and trilinear scalar couplings to be at the light scale. Scenarios in which this arises naturally will be described in

Sec. 5. Assuming this to be true, and further neglecting the $\operatorname{Tr}\left[Y m^{2}\right]$ term, we find that the scalar masses renormalized by the top Yukawa satisfy

$$
\frac{d \mathbf{m}^{2}}{d t}=\frac{h_{t}^{2}}{4 \pi} \mathbf{X}_{\mathrm{low}} \mathbf{m}^{2}
$$

where

$$
\mathbf{X}_{\text {low }}=-\left(\begin{array}{ccc}
3 & 3 & 3 \\
2 & 2 & 2 \\
1 & 1 & 1
\end{array}\right)
$$

and $\mathbf{m}^{\mathbf{2}}=\left(m_{H_{u}}^{2}, m_{U_{3}}^{2}, m_{Q_{3}}^{2}\right)^{T}$. Two eigenvectors of $\mathbf{X}_{\text {low }}$ have eigenvalue 0 ; the third, $\hat{\mathbf{m}}^{\mathbf{2}}=(3,2,1)^{T}$, has eigenvalue -6 . Arbitrary boundary conditions may be evolved 
by first decomposing them along the three eigenvectors [17]. The components parallel to the eigenvectors with zero eigenvalue are constants of the evolution, and the component parallel to $\hat{\mathbf{m}}^{2}$ is asymptotically damped to zero. If the initial conditions are dominated by their $\hat{\mathbf{m}}^{\mathbf{2}}$ component, the three scalar masses will have, subject to the assumptions above, simultaneous fixed points at zero, thereby creating a scalar mass hierarchy. Large components along the other two eigenvectors are not allowed as they lead either to tachyons or large and negative $m_{H_{u}}^{2}$.

To determine whether the fixed points for the mass parameters are reached rapidly enough, let us consider scenarios in which the top quark Yukawa is near its quasifixed point (QFP) [18]. In the low $\tan \beta \mathrm{QFP}$ scenario, $h_{t}$ is drawn to its QFP value of $h_{t}^{\mathrm{FP}} \approx 1.1$ in the infra-red, irrespective of its value at the high scale, as long as this value is not too small. Weak scale parameters are therefore insensitive to the exact value of the top Yukawa at the high scale, which is attractive because our scenario may then be realized without postulating specific and possibly complicated relations between the parameters of the Yukawa and SSB sectors. Given the relation $h_{t}\left(m_{t}\right) \simeq(0.95 / \sin \beta)\left(m_{t}^{\text {pole }} / 175 \mathrm{GeV}\right)$, we find $\tan \beta_{\mathrm{FP}} \approx 1.8$ for the low $\tan \beta \mathrm{QFP}$ scenario. . $^{3}$ Such low values of $\tan \beta$ are currently probed in Higgs boson searches.

In the QFP scenario, it is possible to solve analytically for the low energy values of the soft scalar masses in terms of the high scale boundary conditions, which we denote by zeroes [20]:

$$
\begin{aligned}
& m_{H_{u}}^{2} \simeq m_{H_{u}}^{2}(0)+0.52 M_{1 / 2}^{2}-3 \Delta m^{2} \\
& m_{H_{d}}^{2} \simeq m_{H_{d}}^{2}(0)+0.52 M_{1 / 2}^{2} \\
& m_{Q_{i}}^{2} \simeq m_{Q_{i}}^{2}(0)+7.2 M_{1 / 2}^{2}-\delta_{i} \Delta m^{2} \\
& m_{U_{i}}^{2} \simeq m_{U_{i}}^{2}(0)+6.7 M_{1 / 2}^{2}-\delta_{i} 2 \Delta m^{2} \\
& m_{D_{i}}^{2} \simeq m_{D_{i}}^{2}(0)+6.7 M_{1 / 2}^{2} \\
& m_{L_{i}}^{2} \simeq m_{L_{i}}^{2}(0)+0.52 M_{1 / 2}^{2} \\
& m_{E_{i}}^{2} \simeq m_{E_{i}}^{2}(0)+0.15 M_{1 / 2}^{2}
\end{aligned}
$$

where

$$
\begin{aligned}
\Delta m^{2} \simeq & \frac{1}{6}\left[m_{H_{u}}^{2}(0)+m_{Q_{3}}^{2}(0)+m_{U_{3}}^{2}(0)\right] r \\
& +M_{1 / 2}^{2}\left(\frac{7}{3} r-r^{2}\right)+\frac{1}{3} A_{0}\left(\frac{1}{2} A_{0}-2.3 M_{1 / 2}\right) r(1-r)
\end{aligned}
$$

and, for simplicity, we have assumed a common gaugino mass $M_{1 / 2}$ and trilinear scalar coupling $A_{0}$ at the high scale, which is identified with the scale of coupling constant unification. The subscript $i$ is a generational index; $\delta_{1}=\delta_{2}=0$ and $\delta_{3}=1$. Finally,

\footnotetext{
${ }^{3}$ We ignore here various subtleties associated with the value of the strong coupling and with finite superpartner radiative corrections to $m_{t}^{\text {pole }}$. These can lead to substantial corrections, but may be absorbed in the relevant value of $\tan \beta_{\mathrm{FP}}$ [19.
} 
the parameter $r=\left[h_{t} / h_{t}^{\mathrm{FP}}\right]^{2} \leq 1$ is a measure of the proximity of the top Yukawa coupling to its QFP value at the weak scale.

From Eq. (7) we see that the large Yukawa coupling $h_{t}$ gives a large negative correction to the $H_{u}, Q_{3}$ and $U_{3}$ scalar masses. It is easy to verify that in the limit of $r \rightarrow 1$ and neglecting $M_{1 / 2}$, the equation $m_{H_{u}}=m_{Q_{3}}=m_{U_{3}}=0$ is solved by the boundary conditions

$$
\left[m_{H_{u}}^{2}(0), m_{U_{3}}^{2}(0), m_{Q_{3}}^{2}(0)\right]=m_{0}^{2}[3,2,1]
$$

as expected. (This relation was also noted in Ref. [21].) That is, even if all scalar masses are at some heavy scale $m_{\text {heavy }} \sim 10 \mathrm{TeV}$, if the constraints of Eq. (9) are satisfied, then $m_{H_{u}}, m_{Q_{3}}$, and $m_{U_{3}}$ are still only $\sim m_{\text {light }}$ in the infra-red. From the form of Eqs. (7) and (8), we see that these conclusions hold, roughly, as long as $1-r \lesssim\left(m_{\text {light }} / m_{\text {heavy }}\right)^{2}$ and deviations from the boundary conditions of Eq. (9) satisfy $\Delta m_{H_{u}}^{2}, \Delta m_{Q_{3}}^{2}, \Delta m_{U_{3}}^{2} \lesssim m_{\text {light }}^{2}$.

The light-heavy hierarchy is, of course, also subject to the constraints discussed in Sec. 2. From Eq. (8), we see that we require $M_{1 / 2}, A_{0} \sim m_{\text {light }}$. In addition, the boundary conditions for $H_{d}$ and the other sfermions are constrained by the requirement $\operatorname{Tr}\left[Y m^{2}\right] \lesssim m_{\text {light }}^{2}$; simple boundary conditions, such as the condition that all of these other scalar masses equal $m_{0}$, may be found to satisfy this constraint. Finally, the zero fixed points of the mass parameters receive the usual two-loop gauge corrections of Eq. (3). Because of large group theoretical factors, the two-loop corrections to the light sfermion masses are always more important than the one-loop Yukawa correction. As noted above, for the light scalar squared masses to remain positive, the negative two-loop corrections above must be compensated by positive gaugino mass contributions. The requirement that there be no tachyons or color-breaking minima demands roughly that $M_{1 / 2} \gtrsim \sqrt{\alpha_{s} / 4 \pi} m_{0}$.

We have confirmed the analytic approximations described above with complete numerical calculations including the two-loop gauge corrections. In Fig. 11 we show the renormalization group evolution of the SSB mass parameters for a representative set of boundary conditions satisfying Eq. (9). Despite multi-TeV values at the high scale boundary, we see that the masses renormalized by the large top Yukawa are quickly driven to the weak scale in the infra-red. The other scalar masses remain at the multi-TeV scale, and we see that a scalar mass hierarchy is generated radiatively. To quantify how generic such results are, we display in Figs. 2 and 3 the regions in parameter space for which phenomenologically-desirable squark masses are obtained. In Fig. 2 the weak scale parameters are obtained from the high scale boundary conditions through one-loop renormalization group equations. In the shaded region, both $Q_{3}$ and $U_{3}$ masses are positive and below $1 \mathrm{TeV}$. Any gaugino mass is possible, as long as it is not so large as to drive the $Q_{3}$ and $U_{3}$ masses above $1 \mathrm{TeV}$. In Fig. 3, the

\footnotetext{
${ }^{4}$ In fact, $A_{0} \sim m_{\text {light }}$ is not required in this example if $r \rightarrow 1$; this will not hold in general, however.
} 


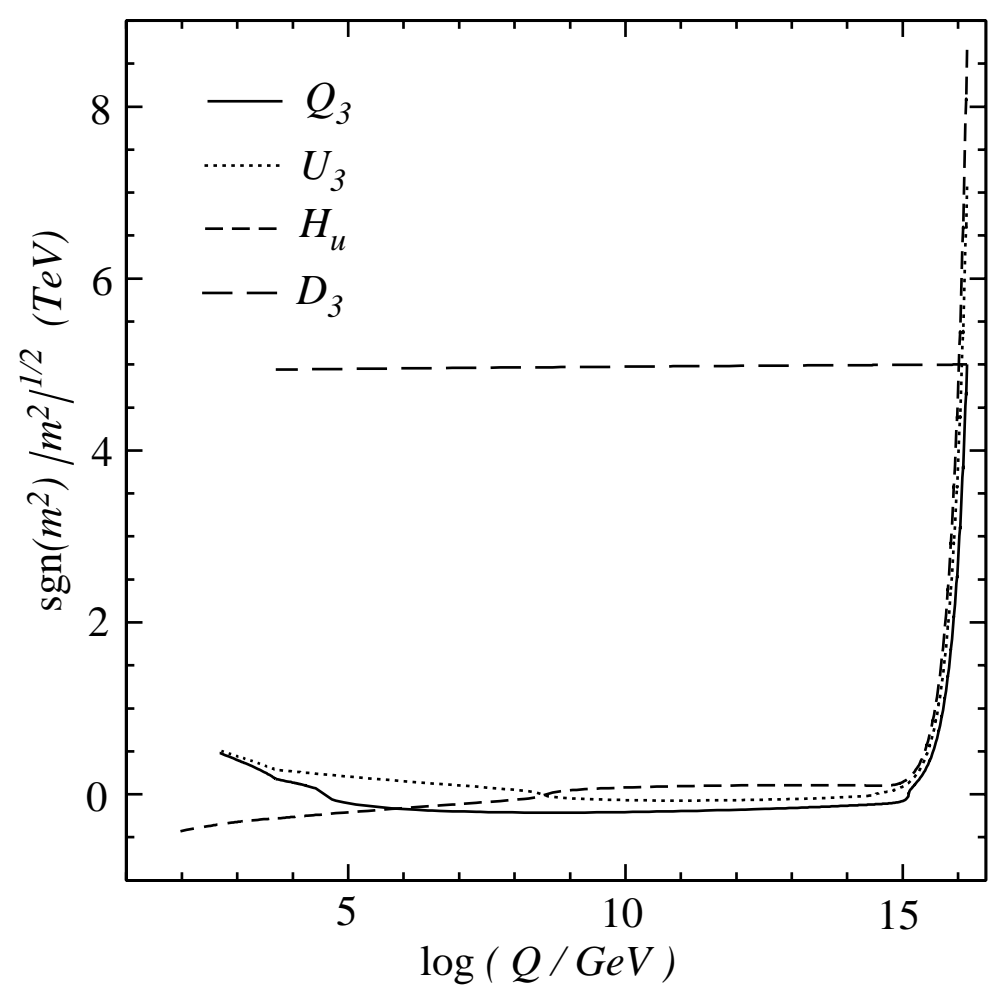

Figure 1: The two-loop renormalization group evolution of SSB masses for a representative case in the low $\tan \beta$ QFP scenario. The boundary conditions at $Q \simeq 2.4 \times 10^{16}$ $\mathrm{GeV}$ are those of Eq. (91) with $m_{0}=5 \mathrm{TeV}$, and $M_{1 / 2}=500 \mathrm{GeV}$ and $A_{0}=0$. The SSB masses for $Q_{3}, U_{3}$, and $H_{u}$ are quickly driven to the weak scale by the top Yukawa coupling, while the rest of the scalars, represented by $D_{3}$ here, remain at the multi-TeV scale.

two-loop gauge contributions are included. As noted above, these contributions must be compensated by gaugino contributions to avoid tachyons and color-breaking minima, and so now, for a given $m_{0}$, there is a minimum allowed $M_{1 / 2}$. We see, however, that there is still a substantial band in which all phenomenological requirements are met, and the $Q_{3}$ and $U_{3}$ masses are below a $\mathrm{TeV}$.

In this scenario, the fine-tuning associated with $m_{H_{u}}$ and the squark fields $Q_{3}$ and $U_{3}$, which are strongly coupled to $H_{u}$, has been successfully eliminated. Unfortunately, $m_{H_{d}}$ is not affected by the Yukawa fixed point in the low $\tan \beta$ scenario and remains at the heavy scale. Electroweak symmetry breaking therefore requires $\mu^{2} \sim m_{H_{d}}^{2} \sim$ $m_{\text {heavy }}^{2}$, and this scenario is still fine-tuned.p

This flaw may be avoided in high $\tan \beta$ scenarios, to which we will turn in the following section. Before doing so, however, we collect here a number of remarks.

\footnotetext{
${ }^{5}$ The requirement that squark mixing not lead to color-breaking minima also leads to the constraint $m_{\text {light }} m_{H_{d}}<m_{Q}^{2} \simeq 7 M_{1 / 2}^{2}$, which is, however, weaker than the constraints discussed above and is easily satisfied.
} 


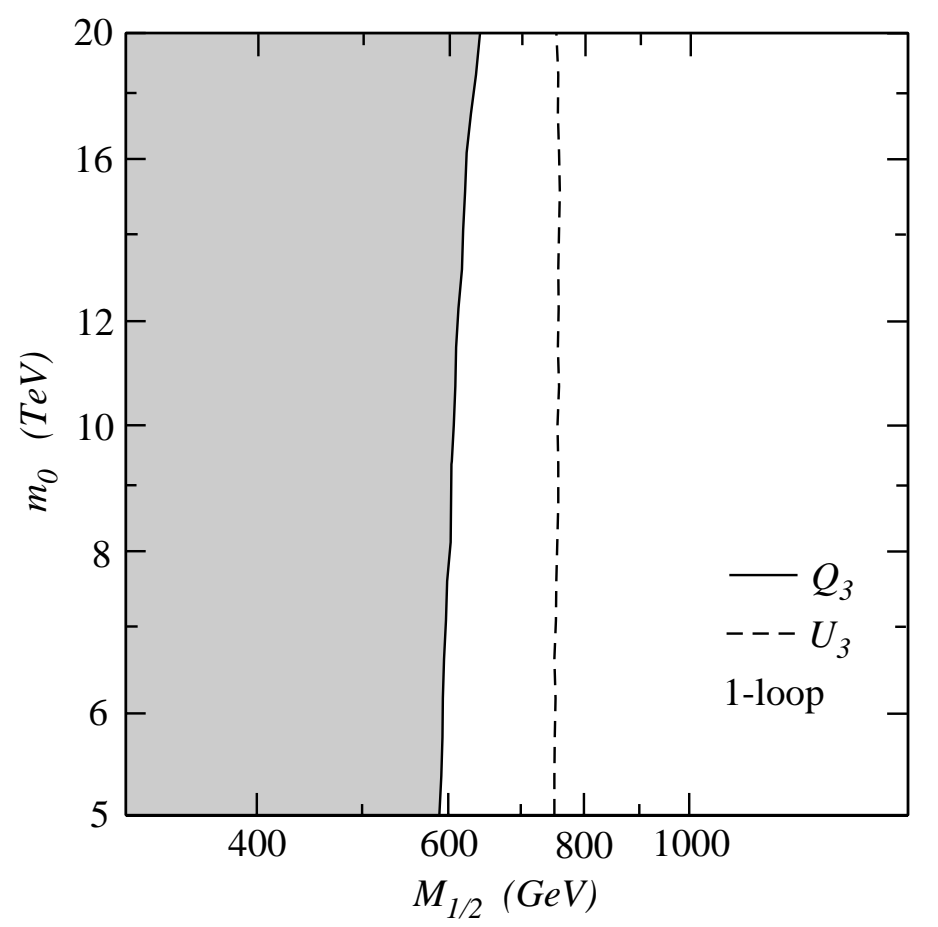

Figure 2: The allowed region (shaded) in the $\left(M_{1 / 2}, m_{0}\right)$ parameter place, where $M_{1 / 2}$ is the high scale gaugino mass, and $m_{0}$ specifies the high scale scalar masses through Eq. (9). One-loop renormalization group equations are used and $A_{0}=0$. To the left of the solid (dashed) contour, the scalar $Q_{3}\left(U_{3}\right)$ mass is below $1 \mathrm{TeV}$ at the weak scale. All physical squared masses are positive and electroweak symmetry is properly broken throughout the plane. Note the different mass scales.

First, note that the boundary conditions of Eq. (9) are inconsistent with any minimal GUT embedding requiring $m_{Q_{3}}(0)=m_{U_{3}}(0)$. Second, large $(\gtrsim 10 \%)$ and negative finite mass corrections to the top quark mass may increase the low $\tan \beta$ QFP value to $\tan \beta^{\mathrm{FP}} \gg 1$. In this case, as is evident from Eq. (西), the fine-tuning related to large $m_{H_{d}}$ is significantly diminished, and $m_{H_{d}} \sim m_{\text {heavy }}$ may be tolerated. However the finite mass contributions realized by supersymmetric QCD corrections in most models are $\lesssim 10 \%$, and so this scenario may be difficult to realize.

Finally, it is entertaining to note that $m_{H_{d}},|\mu| \gg m_{\text {weak }}$ is actually preferred by coupling constant unification, as it leads to a pattern of superparticle threshold corrections that diminishes the prediction for the strong coupling $\alpha_{s}\left(m_{\text {weak }}\right)$. In the absence of threshold corrections, one predicts too-large $\alpha_{s}\left(M_{Z}\right) \approx 0.13$, and most typical patterns of superparticle threshold corrections only aggravate this problem. 


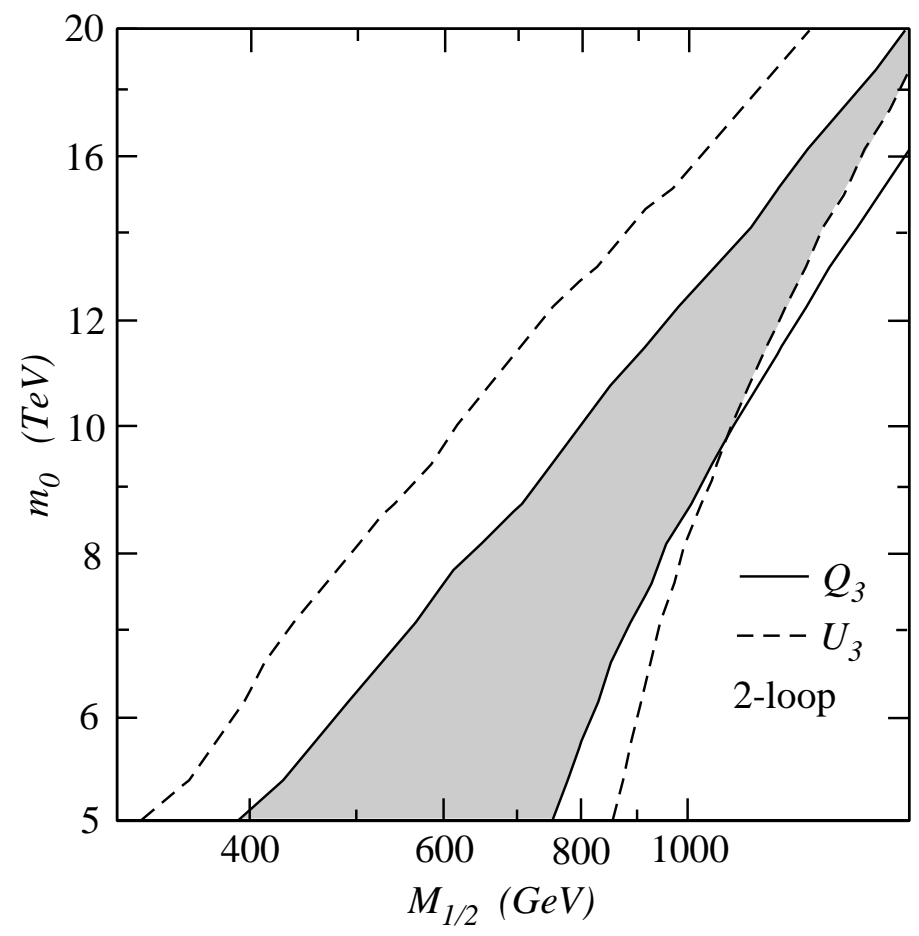

Figure 3: Same as in Fig. 2, but including the two-loop gauge contributions in the evolution. To the left of the left-most solid (dashed) contour, the scalar $Q_{3}\left(U_{3}\right)$ mass is negative at the weak scale; to the right of the right-most solid (dashed) contour, the scalar $Q_{3}\left(U_{3}\right)$ mass is above $1 \mathrm{TeV}$. In the shaded region, both masses are positive and below $1 \mathrm{TeV}$.

\section{Radiative Hierarchy with High $\tan \beta$}

The fine-tuning situation may be resolved in the case of high $\tan \beta \sim 50-60$, where both $h_{t}$ and $h_{b} \equiv h_{d}^{33}$ are near their fixed points. The coupled set of renormalization group equations is now more complicated. However, assuming $h_{t} \approx h_{b}$, and neglecting for simplicity all gaugino masses, trilinear scalar couplings and $\operatorname{Tr}\left[\mathrm{Ym}^{2}\right]$ as before, we find that the scalar masses evolve as

$$
\frac{d \mathbf{m}^{2}}{d t}=\frac{h_{t}^{2}}{4 \pi} \mathbf{X}_{\mathrm{high}} \mathbf{m}^{2}
$$

where

$$
\mathbf{X}_{\text {high }}=-\left(\begin{array}{ccccc}
3 & 3 & 3 & 0 & 0 \\
2 & 2 & 2 & 0 & 0 \\
1 & 1 & 2 & 1 & 1 \\
0 & 0 & 2 & 2 & 2 \\
0 & 0 & 3 & 3 & 3
\end{array}\right)
$$


and here $\mathbf{m}^{\mathbf{2}}=\left(m_{H_{u}}^{2}, m_{U_{3}}^{2}, m_{Q_{3}}^{2}, m_{D_{3}}^{2}, m_{H_{d}}^{2}\right)^{T}$. Three eigenvectors of $\mathbf{X}_{\text {high }}$ have eigenvalue 0 ; the other two are $\hat{\mathbf{m}}_{1}^{2}=(-3,-2,0,2,3)^{T}$ with eigenvalue -5 and $\hat{\mathbf{m}}_{\mathbf{2}}^{\mathbf{2}}=$ $(3,2,2,2,3)^{T}$ with eigenvalue -7 . We thus expect a two parameter family of boundary conditions leading to a scalar mass hierarchy.

As before, we consider the QFP framework, but now for high $\tan \beta$. Neglecting gaugino masses and the trilinear scalar couplings, we find simple solutions for the low-energy masses in terms of their high scale boundary values [22]:

$$
\begin{aligned}
m_{H_{u}}^{2} & \simeq m_{H_{u}}^{2}(0)-3 \Delta m_{t}^{2} \\
m_{H_{d}}^{2} & \simeq m_{H_{d}}^{2}(0)-3 \Delta m_{b}^{2} \\
m_{Q_{i}}^{2} & \simeq m_{Q_{i}}^{2}(0)-\delta_{i}\left(\Delta m_{t}^{2}+\Delta m_{b}^{2}\right) \\
m_{U_{i}}^{2} & \simeq m_{U_{i}}^{2}(0)-\delta_{i} 2 \Delta m_{t}^{2} \\
m_{D_{i}}^{2} & \simeq m_{D_{i}}^{2}(0)-\delta_{i} 2 \Delta m_{b}^{2} \\
m_{L_{i}}^{2} & \simeq m_{L_{i}}^{2}(0) \\
m_{E_{i}}^{2} & \simeq m_{E_{i}}^{2}(0)
\end{aligned}
$$

where

$$
\begin{aligned}
\Delta m_{t}^{2} & \simeq \frac{1}{7}\left[m_{H_{u}}^{2}(0)+m_{Q_{3}}^{2}(0)+m_{U_{3}}^{2}(0)\right] r \\
& -\frac{1}{10}\left[m_{H_{u}}^{2}(0)-m_{H_{d}}^{2}(0)+m_{U_{3}}^{2}(0)-m_{D_{3}}^{2}(0)\right]\left[\frac{5}{7} r+(1-r)^{\frac{5}{7}}-1\right] \\
\Delta m_{b}^{2} & \simeq \frac{1}{7}\left[m_{H_{d}}^{2}(0)+m_{Q_{3}}^{2}(0)+m_{D_{3}}^{2}(0)\right] r \\
& +\frac{1}{10}\left[m_{H_{u}}^{2}(0)-m_{H_{d}}^{2}(0)+m_{U_{3}}^{2}(0)-m_{D_{3}}^{2}(0)\right]\left[\frac{5}{7} r+(1-r)^{\frac{5}{7}}-1\right]
\end{aligned}
$$

and, as before, $r=\left[h_{t} / h_{t}^{\mathrm{FP}}\right]^{2} \leq 1$. In this solution, we have neglected small differences in the top and bottom Yukawa coupling evolution, and assumed vanishing leptonic couplings. In particular, we neglect $h_{\tau}$; see the discussion below.

In contrast to the previous low $\tan \beta$ case, $h_{b}$ is now significant. We must then demand that the $H_{u}, H_{d}, Q_{3}, U_{3}$, and $D_{3}$ scalar masses all be driven to zero in the infra-red. We find that this scenario is obtained for an extremely simple twoparameter family of boundary conditions given by

$$
\begin{aligned}
m_{H_{u}}^{2}(0) & =\frac{3}{2} m_{U_{3}}^{2}(0) \\
m_{H_{d}}^{2}(0) & =\frac{3}{2} m_{D_{3}}^{2}(0) \\
m_{Q_{3}}^{2}(0) & =\frac{1}{2}\left[m_{U_{3}}^{2}(0)+m_{D_{3}}^{2}(0)\right] .
\end{aligned}
$$

These boundary conditions are just a reparametrization of the space spanned by the eigenvectors $\hat{\mathbf{m}}_{\mathbf{1}}^{2}$ and $\hat{\mathbf{m}}_{\mathbf{2}}^{2}$. Clearly, in this case both Higgs mass parameters are affected 
by the fixed point and no fine-tuning is required (aside from the moderate tuning at the level of $m_{W}^{2} / m_{\text {light }}^{2}$, which is always associated with such high values of $\tan \beta$ [24]).

The solutions of Eq. (12) are valid for boundary values $h_{\tau}^{2}(0) \ll h_{b}^{2}(0)$ and $h_{\tau} \ll$ $4 \pi m_{\text {light }} / m_{\text {heavy }}$. The first relation is found in a certain range of very high $\tan \beta \sim$ $50-60$.9] (See, for example, Fig. 1 of Ref. [19].) One often associates the high $\tan \beta$ QFP scenario with either $h_{b}=h_{\tau}$ or $h_{t}=h_{b}=h_{\tau}$ unification at the GUT scale, as implied by minimal $\mathrm{SU}(5)$ and $\mathrm{SO}(10)$ GUTs, respectively. A subset of the solutions of Eq.(15), with $m_{Q_{3}}^{2}(0)=m_{U_{3}}^{2}(0)=m_{D_{3}}^{2}(0)$ and $m_{H_{u}}^{2}(0)=m_{H_{d}}^{2}(0)=$ $\frac{3}{2} m_{Q}^{2}(0)$, is consistent with such a GUT embedding. (The hypercharge trace condition is automatically satisfied in this case.) In general, however, the boundary conditions need not admit a true (minimal) GUT embedding, and we therefore do not require such scalar mass relations or the accompanying Yukawa coupling unifications.

It is difficult to incorporate analytically the effects of a non-negligible $h_{\tau}$, and generally an involved numerical analysis is required. In Ref. [22] this effect was estimated, but the results were valid only for $0.6 \lesssim r \lesssim 0.95$. The required boundary conditions have a complicated dependence on $r$ and therefore do not have obviously simple forms away from the QFP value of $r=1$.

In the quantitative discussions above, we have focused on only two simple scenarios with minimal field content. It should be stressed, however, that while the required boundary conditions depend on the specific Yukawa fixed point structure, the existence of such boundary conditions stems from the general existence of such a structure. Hence, it is reasonable to speculate that our observations apply more generally. For example, one could look for similar QFP solutions in the MSSM extended by a gauge singlet $S$ interacting through the superpotential term $S H_{u} H_{d}$, or at the case of lepton number violating Yukawa couplings with simultaneous fixed points [23]. Many other such examples are possible.

\section{$5 \quad$ High-energy Frameworks and $R$ Symmetry}

We have seen that inverted scalar hierarchies may be generated radiatively for certain boundary conditions. Such boundary conditions are both highly constrained and highly predictive, and it is of some interest to investigate specific high energy frameworks that give such mass patterns. Here we will limit ourselves to a discussion of general principles that lead to the required features.

Let us concentrate on the high $\tan \beta$ scenario. The appearance of a light-heavy hierarchy in the scalar mass sector can only occur if there is already a hierarchy between the scalar masses (heavy) and the $\mu$ parameter, $m_{3}^{2}$, gaugino masses, and $A$ terms (light). (Electroweak symmetry breaking requires $m_{3}^{2}$ at the light scale, since $m_{3}^{2}=\frac{1}{2}\left(m_{H_{u}}^{2}+m_{H_{d}}^{2}+2 \mu^{2}\right) \sin 2 \beta$. $)$

\footnotetext{
${ }^{6}$ Again, the exact value of $\tan \beta$ for which $h_{t}(0) \simeq h_{b}(0) \gg h_{\tau}(0)$ depends sensitively on lowenergy finite radiative corrections to the $t$ and $b$-quark masses and on the exact value of the strong coupling.
} 
Such a hierarchy might be generated by an approximate U(1) symmetry. In the absence of the $\mu$ and SSB parameters, the MSSM possesses two global U(1) symmetries: a Peccei-Quinn symmetry, under which all components of a given superfield have the same charge, and an $R$ symmetry, under which the boson and fermion components of a given superfield differ by one unit. If $\mu$ and the SSB parameters are viewed as spurion fields [26], we may choose the following charge assignments for them: $P Q(\mu)=P Q\left(m_{3}^{2}\right)=1$, with all others PQ neutral, $R\left(m_{0}\right)=R(\mu)=0$, and $R\left(m_{3}^{2}\right)=R\left(M_{1 / 2}\right)=R(A)=-2$. Under suitable linear combinations of these two symmetries, such as $R+P Q$, all parameters are charged, except for the scalar masses $m_{0}$. (Note that scalar masses are neutral, and gaugino masses and $A$-terms are charged, for all possible linear combinations.) Thus, an approximate U(1) symmetry, such as an $R+P Q$ symmetry, naturally produces the necessary hierarchy, and the presence of $R$ symmetries may play a vital role in realizing models that exhibit the inverted hierarchy.

Alternatively, the suppression of the necessary parameters may be the result of some other mechanism. Assume, for example, that the scale at which SUSY-breaking is communicated to the MSSM, $M$, is significantly higher than the initial scale of SUSY-breaking itself, $\sqrt{F}$. We can then express the most general set of operators in an expansion in powers of $\sqrt{F} / M$. The leading terms in that expansion that generate the $\mu$ parameter and soft terms have the following form:

$$
\begin{aligned}
\text { Scalar masses : } & \int d^{4} \theta \Phi_{i}^{\dagger} \Phi_{i}\left[\frac{S^{\dagger} S}{M^{2}}+\frac{Z^{\dagger} Z}{M^{2}}+\cdots\right] \\
\mu \text { parameter : } & \int d^{4} \theta H_{u} H_{d}\left[\frac{S^{\dagger}}{M}+\cdots\right] \\
\text { Gaugino masses : } & \int d^{2} \theta W^{\alpha} W_{\alpha}\left[\frac{S}{M}+\cdots\right] \\
A \text {-terms : } & \int d^{2} \theta \Phi_{i} \Phi_{j} \Phi_{k}\left[\frac{S}{M}+\cdots\right] \text { and } \int d^{4} \theta \Phi_{i}^{\dagger} \Phi_{i}\left[\frac{S}{M}+\cdots\right],
\end{aligned}
$$

where the $W_{\alpha}$ are gauge vector supermultiplets containing the standard model gauginos, the $\Phi_{i}$ are standard model chiral superfields, and $S$ and $Z$ represent SUSYbreaking gauge singlet and non-singlet superfields, respectively. These terms give SSB parameters and the $\mu$ parameter when the $S$ and $Z$ fields get $F$-term vacuum expectation values: $S \rightarrow F_{S} \theta^{2}, Z \rightarrow F_{Z} \theta^{2}$ (and, in the second source for $A$-terms, $\left.\Phi_{i}^{\dagger} \rightarrow F_{\Phi_{i}}^{*} \bar{\theta}^{2} \sim \Phi_{j} \Phi_{k} \bar{\theta}^{2}\right)$

From the expressions above, it is clear that the terms corresponding to dimension 3 operators rely on SUSY-breaking singlet fields at leading order in $M^{-1}$, while the scalar masses do not. Therefore, in any scenario in which $F_{S} \ll F_{Z}$ (or $S$ is absent from the spectrum), $\mu$, the gaugino masses, and all $A$-terms will be suppressed relative to scalar masses. For example, to generate the desired hierarchy, it is sufficient for $F_{S}$ to be generated radiatively so that $F_{S} \sim \alpha / 4 \pi F_{Z}$. (Note, however, that $m_{3}^{2}$ must be suppressed by some other means, such as the U(1) symmetries discussed above.) 
Finally, it is interesting to ask whether such a hierarchy could ever occur in supergravity-mediated SUSY-breaking models. It is known that in models without singlets, gaugino masses are suppressed relative to scalar masses. If we further assume that there are no Planck scale vacuum expectation values in the hidden sector (as is expected in models that break SUSY in the flat limit), then $A$-terms will also be suppressed [27]. In such scenarios, conventional contributions to the gaugino masses and $A$-terms are highly suppressed, and the dominant contributions have recently been shown to be those arising from the superconformal anomaly [28]. In fact, the natural suppression of gaugino masses relative to squark masses is then one-loop, roughly corresponding to the size we require in our mechanism.

Once the hierarchy between the scalar and gaugino masses is generated, it is still necessary to understand the particular form of the scalar mass boundary conditions that are required in these scenarios.

The rational relations that are required among the soft masses in the previous sections are immediately reminiscent of the relations one would expect were soft masses to be communicated via $D$-terms of broken gauge symmetries. This results in terms $\mathcal{L}=\frac{g^{2}}{2}\left[\operatorname{Tr} Q_{i} m_{i}^{2} \pm \xi\right]^{2}$, where $Q$ is a charge in the Cartan subalgebra of the broken group and $\xi$ is an order parameter of the group's breaking. Note that the squared masses are always proportional to the broken Cartan charges of the fields. Unfortunately, it is impossible to have the spectrum of charges corresponding to Eq. (9) [Eq. (15)] and simultaneously demand invariance of the top [top and bottom] Yukawa coupling under the broken symmetry group, which is a natural assumption given its large size.

One might also consider the framework of weakly coupled string theory. There, the dilaton field is one of the singlets $S$ above. If SUSY-breaking is dominated by the dilaton $F$ component, then gaugino masses and (universal) scalar masses are of the same order [29]. However, if SUSY-breaking is dominated by $F$ components of moduli $\Phi_{M}$, gaugino masses arise only at loop-level in string theory, giving $M_{1 / 2} \sim$ $\left(\alpha_{\text {string }} / 4 \pi\right) m_{0}$. (Of course, a mechanism for suppressing the $A$-terms is also needed.) Incidentally, in the moduli-dominated scenario, scalar masses are generically all at the same scale, but may differ by order one coefficients given by the Kähler metric:

$m_{i}^{2} \approx K_{i i} m_{0}^{2}$. This is exactly the necessary condition for radiative inverted hierarchy generation. In this framework, the boundary condition scalar mass ratios that we derived above correspond to ratios of modular weights of the different fields.

\section{The $C P$ and Polonyi Problems}

The $R$ symmetry discussed above was previously studied in Ref. [25], where a number of attractive phenomenological features were noted. In that work, an approximate $R$ symmetry was seen as a possible source for a hierarchy $\mu, M_{1 / 2}, A \sim 1 \mathrm{GeV} \ll m_{0} \sim$ $m_{\text {weak }}$. In this study, we are considering mass scales roughly 100 times those discussed there. However, as most of the attractive features discussed there result from the hier- 
archy itself, they apply equally well here. For example, supersymmetric contributions to electron and neutron electric dipole moments are $d_{e, n} \propto\left(1 / m_{0}^{2}\right)\left(M_{1 / 2} \tilde{m} / m_{0}^{2}\right)$, where $\tilde{m} \sim \mu, A$, and $m_{0}$ represents scalar masses of the first generation. These contributions are therefore suppressed both by the large scalar mass scale and by the hierarchy between $m_{\text {light }}$ and $m_{\text {heavy }}$, and are well below current experimental bounds.

Our models also have an important cosmological virtue. Many supergravity models contain a boson $\phi$, the Polonyi field, with mass of order the gravitino mass. The Polonyi field has gravitational couplings and, consequently, an extremely long lifetime $\tau \sim M_{P}^{2} / m_{\phi}^{3}$, where $M_{P}$ is the Planck mass. For such models with gravitino masses of order $100 \mathrm{GeV}$, the Polonyi field typically decays during or after temperatures of order $1 \mathrm{MeV}$, thereby potentially ruining nucleosynthesis. This is often referred to as the "Polonyi problem" [30], and is a serious cosmological difficulty for many models.

The Polonyi problem may be solved, for example, in particular SUSY-breaking scenarios [31]. Irrespective of the SUSY-breaking mechanism, however, in the models discussed here, the Polonyi problem is always alleviated, as the gravitino mass $m_{3 / 2} \sim$ $m_{0}$ is in the multi-TeV range. It has been pointed out that this provides a solution to the Polonyi problem, since in this case even a Polonyi field with mass $m_{\phi} \sim m_{3 / 2} \sim 10$ $\mathrm{TeV}$ decays sufficiently quickly to avoid the difficulty mentioned above [32. Potential problems with generating the baryon asymmetry and overclosing the universe with Polonyi decay products may also be solved, the first with Affleck-Dine baryogenesis, and the second with the presence of a very light and stable superpartner or with $R$-parity violation 33 .

\section{Summary and Outlook}

To conclude, we have investigated the possibility that soft SUSY-breaking scalar mass parameters are not $\lesssim 1 \mathrm{TeV}$ at some high scale boundary, as is typically assumed, but rather, are all in the multi-TeV range. For particular boundary conditions, given in Eqs. (9) and (15), we find that scalars with large Higgs couplings are asymptotically driven to the weak scale by renormalization group evolution, while the remaining scalars stay at the multi-TeV scale. By this mechanism, the light scalars are precisely those that must be light to preserve the gauge hierarchy, and the heavy scalars are precisely those corresponding to light fermions that must be heavy to satisfy stringent flavor-changing constraints.

As in all models with hierarchical squark masses, it is important to note that multiTeV scalar masses by themselves do not completely satisfy all flavor constraints [15, [16. In the above analysis, we have discussed only the evolution of the flavor diagonal masses. However, it is possible that off-diagonal masses are present at the high scale; such masses are largely unaffected by renormalization group evolution. Recent improvements in calculations of $K^{0}-\bar{K}^{0}$ mixing have strengthened this most stringent constraint, so that now, even with $m_{\text {heavy }} \sim 10 \mathrm{TeV}$, the off-diagonal squark masses must roughly satisfy $m_{12}^{2} / m_{\text {heavy }}^{2} \lesssim 0.1$ [1]. This requirement on mixings (or non- 
degeneracies) is, however, relatively mild and is a great improvement over analogous constraints on models with squarks below the $\mathrm{TeV}$ scale.

There are several experimental signatures of these models. As evident from the discussion above, detectable effects in the kaon system are possible. In addition, although the requirement of no tachyons implies roughly $m_{13}^{2}, m_{23}^{2} \lesssim m_{\text {light }} m_{\text {heavy }}$, large effects in the $B$ system, for example, may be possible, and are potentially observable at current of near-future experiments [8]. There are also implications for the high energy frontier. At least some gauginos and some third generation sfermions are predicted to be accessible at the next generation of collider experiments. While the $m_{\text {heavy }}$ sector will not be, it may then be explored indirectly by measurements of the superoblique corrections of Ref. [9]. Although very massive scalars decouple from many observables, they leave their imprint on low energy processes by breaking the equality of gauge boson-fermion-fermion couplings and the corresponding gauginofermion-sfermion couplings. These deviations are non-decoupling. The superoblique parameters are therefore sensitive to arbitrarily heavy MSSM sfermions, and may be measured to high accuracy in processes involving the observable superparticles [9, 10].

In this scenario, several requirements must be met. First, the flavor off-diagonal masses discussed above must be suppressed relative to flavor diagonal ones. Of course, as noted above, the necessary suppressions are mild relative to models with all scalars below the TeV scale. It is also worth noting that in such conventional models, even if some mechanism for suppressing flavor violation is implemented, (flavor-conserving) constraints on electric dipole moments and the Polonyi problem may still be rather severe; as argued in Sec. 6, these problems are naturally alleviated in the models discussed here.

In addition, the requirement of extreme scalar degeneracy or alignment to remove dangerous flavor-changing contributions is replaced by the requirement of particular high scale boundary conditions. In the absence of a more fundamental theory, this is not an obvious improvement. However, this scenario opens a new arena for SUSY model building. With regard to the supersymmetric flavor problem, it presents the possibility that a solution is provided by some dynamical mechanism that produces the required boundary conditions, such as the simple conditions of Eq. (15). We have discussed theoretical motivations for the required hierarchies and a possible relation to $R$ symmetries. More generally, and independent of the SUSY flavor problem, it raises the possibility of scenarios in which electroweak symmetry breaking is not finetuned, even though the fundamental scale for the soft SUSY-breaking parameters is $\sim 10 \mathrm{TeV}$, rather than $\sim 1 \mathrm{TeV}$ as is typically assumed.

Finally, we note that, while our illustrations have been limited to the MSSM, these observations should apply more generally. It would be particularly interesting to pursue this framework in models with extended fixed point structures, and also more extensively in the high $\tan \beta$ regime. 


\section{Acknowledgments}

The authors thank J. Bagger, P. G. O. Freund, T. Moroi and A. Pomarol for stimulating comments and conversations. JLF is grateful to the theory groups of Stanford and SLAC for hospitality, and NP thanks the theory group at CERN for its hospitality. JLF is supported by the Department of Energy under contract DE-FG0290ER40542 and through the generosity of Frank and Peggy Taplin. CK is supported by the Department of Energy under contract DE-AC03-76SF00098. The work of NP

is supported by the NSF under grant NSF-PHY-94-23002 and by the Department of Energy under contract DE-FG02-96ER40559.

\section{References}

[1] For recent quantitative analyses, see M. Ciuchini et al., hep-ph/9808328; R. Contino and I. Scimemi, hep-ph/9809437.

[2] S. Dimopoulos and G. F. Giudice, Phys. Lett. B357, 573 (1995); A. Pomarol and D. Tommasini, Nucl. Phys. B466, 3 (1996).

[3] G. Dvali and A. Pomarol, Phys. Rev. Lett. 77, 3728 (1996); ibid., Nucl. Phys. B522, 3 (1998).

[4] A. G. Cohen, D. B. Kaplan, and A. E. Nelson, Phys. Lett. B388, 588 (1996).

[5] R. N. Mohapatra and A. Riotto, Phys. Rev. D55, 1 (1997); R.-J. Zhang, Phys. Lett. B402, 101 (1997); A. E. Nelson and D. Wright, Phys. Rev. D56, 1598 (1997). J. Hisano, K. Kurosawa, and Y. Nomura, hep-ph/9810411.

[6] H. P. Nilles and N. Polonsky, Phys. Lett. B412, 69 (1997); D. E. Kaplan, F. Lepeintre, A. Masiero, A. E. Nelson, and A. Riotto, hep-ph/9806430.

[7] S. Ambrosanio and A. E. Nelson, Phys. Lett. B411, 283 (1997).

[8] A. G. Cohen, D. B. Kaplan, F. Lepeintre, and A. E. Nelson, Phys. Rev. Lett. 78, 2300 (1997).

[9] H.-C. Cheng, J. L. Feng, and N. Polonsky, Phys. Rev. D56, 6875 (1997); ibid., 57, 152 (1998).

[10] K. Hikasa and Y. Nakamura, Zeit. für Physik C70, 139 (1996); ibid., 71, 356 (1996); M. M. Nojiri, K. Fujii, and T. Tsukamoto, Phys. Rev. D54, 6756 (1996); M. M. Nojiri, D. M. Pierce, and Y. Yamada, Phys. Rev. D57, 1539 (1998); S. Kiyoura, M. M. Nojiri, D. M. Pierce, and Y. Yamada, Phys. Rev. D58, 075002 (1998); E. Katz, L. Randall, and S.-F. Su, hep-ph/9801416. 
[11] Preliminary results of our investigation were reported in N. Polonsky, Nucl. Phys. Proc. Suppl. 62, 204 (1998).

[12] See, for example, I. Jack and D. R. T. Jones, Phys. Lett. B349, 294 (1995); I. Jack, D. R. T. Jones, and K. L. Roberts, Nucl. Phys. B455, 83 (1995); P. M. Ferreira, I. Jack, and D. R. T. Jones, Phys. Lett. B357, 359 (1995); M. Lanzagorta and G. G. Ross, Phys. Lett. B364, 163 (1995).

[13] M. Drees, Phys. Rev. D33, 1468 (1986).

[14] S. P. Martin and M. T. Vaughn, Phys. Rev. D50, 2282 (1994); I. Jack, D. R. T. Jones, Phys. Lett. B333, 372 (1994); Y. Yamada, Phys. Rev. D50, 3537 (1994).

[15] N. Arkani-Hamed and H. Murayama, Phys. Rev. D56, 6733 (1997).

[16] K. Agashe and M. Graesser, hep-ph/9801446; D. Wright, hep-ph/9801449.

[17] See, for example, M. Wise, in Proceedings of TASI '87, edited by R. Slansky and G. West (World Scientific, Singapore, 1988); J. Bagger, in Proceedings of TASI '95, edited by D. Soper (World Scientific, Singapore, 1996), pp. 109-162.

[18] C. T. Hill, Phys. Rev. D24, 691 (1981).

[19] N. Polonsky, Phys. Rev. D54, 4537 (1996), and references therein.

[20] L. E. Ibanez and C. Lopez, Nucl. Phys. B233, 511 (1984); M. Carena, M. Olechowski, S. Pokorski, and C. E. M. Wagner, Nucl. Phys. B419, 213 (1994); M. Carena, and C. E. M. Wagner, Nucl. Phys. B452, 45 (1995).

[21] M. Carena, P. Chankowski, M. Olechowski, S. Pokorski, and C. E. M. Wagner, Nucl. Phys. B491, 103 (1997).

[22] M. Carena and C. E. M. Wagner, hep-ph/9407209.

[23] V. Barger, M. S. Berger, R. J. N. Phillips, and T. Wohrmann, Phys. Rev. D53, 6407 (1996).

[24] See, for example, R. Rattazzi and U. Sarid, Phys. Rev. D53, 1553 (1996).

[25] J. L. Feng, N. Polonsky, and S. Thomas, Phys. Lett. B370, 95 (1996).

[26] S. Dimopoulos and S. Thomas, Nucl. Phys. B465, 23 (1996).

[27] I. Joichi and M. Yamaguchi, Phys. Lett. B342, 111 (1995).

[28] L. Randall and R. Sundrum, hep-th/9810155; G. Giudice, M. Luty, H. Murayama, and R. Rattazzi, hep-ph/9810442. 
[29] See, for example, J. Louis and Y. Nir, Nucl. Phys. B447, 18 (1995), and references therein.

[30] G. D. Coughlan, W. Fischler, E. W. Kolb, S. Raby, and G. G. Ross, Phys. Lett. B131, 59 (1983).

[31] See, for example, T. Banks, D. B. Kaplan, and A. E. Nelson, Phys. Rev. D49, 779 (1994); M. Dine, L. Randall, and S. Thomas, Phys. Rev. Lett. 75, 398 (1995); ibid., Nucl. Phys. B458, 291 (1996).

[32] J. Ellis, D. V. Nanopoulos, and M. Quiros, Phys. Lett. B174, 176 (1986).

[33] T. Moroi, M. Yamaguchi, and T. Yanagida, Phys. Lett. B342, 105 (1995); M. Kawasaki, T. Moroi, and T. Yanagida, Phys. Lett. B370, 52 (1996); and references therein. 